\title{
NONLINEAR MULTIPLE-SCALE SOLUTION OF A CYLINDRICAL SHELL*
}

\author{
BY
}

\author{
JAMES R. STAFFORD AND ADOLF T. HSU \\ West Virginia University, Morgantown
}

\begin{abstract}
A multiple-scale perturbation technique is used with a two-parameter expansion to study the asymptotic solution of Reissner's axisymmetric finite-deformation equations for a circular cylindrical shell with an edge-bending moment load. Beyond the assumptions of Reissner's differential equations, it is assumed that (1) the rotations of a shell element are finite but not excessively large, (2) thickness variations in the differential equations are of order one and (3) the boundary-layer behavior is of the linear bending type to a first approximation. An asymptotic solution is then found which is uniformly valid in that it contains boundary-layer effects and corrections for extending the analysis into the shell's interior. Upon considering certain limits, it is observed that the solution contains well-established linear and nonlinear approximations to the solution.

1. Introduction. The analysis presented herein involves the use of the multiplescale method of perturbation [1,2] to solve the nonlinear deformation problem of a thin circular cylindrical shell with an edge-bending moment load. The multiple-scale method of solution has been used [3] to find the solution to the homogeneous linear differential equations governing variable-thickness circular cylindrical shells undergoing axisymmetric deformation. The present solution is based on approximations to Reissner's nonlinear finite-deformation equations [4] for an isotripic elastic shell and includes the effect of gradual changes in thickness.
\end{abstract}

Heuristics leading to the solution of differential equations by the method of multiple scales have been advanced by Cochran [2] and by Cole and Kevorkian [5]. The analysis is based on the observation that the composite solution is dependent upon two coordinates, one of which is of order one in the boundary layer while the other is of order one in the outer region. The effect that each of these coordinates has on the governing differential equations is realized through the ingenious multiple-scale transformation which converts ordinary differential equations into partial differential equations. Arbitrary functions are found on the basis of "Lighthill's principle" of limiting the singularity of the solution. A brief discussion of this method of analysis is found in Van Dyke [1].

A linear asymptotic solution for variable-thickness shells of revolution has been found by Hildebrand [6]. Hildebrand generates his solution by expanding the dependent variables in terms of a small geometric parameter. Also, special thickness variations have bcen considered [7] for linear shell problems.

Some nonlinear effects of edge loading have been considered [8,9]. Of particular interest is Reissner's solution [9] using an asymptotic expansion in terms of load-depen-

* Received June 7, 1971. This work was completed under sponsorship of the National Science Foundation (Grant GK-5004). 
dent perturbation parameters to solve the nonlinear boundary layer equations. Although a useful result, Reissner's expansion is also restricted to very thin shells, since he excluded the geometric parameter dependent upon thickness-to-curvature ratios.

In the present contribution to the literature on edge-loaded shells, Reissner's method of scaling the variables $[8,9]$ is used to obtain a form of the differential equations which, to a first approximation, yield linear bending terms in the boundary layer. Then, extending the considerations beyond the first approximation, a two-parameter expansion is incorporated with the multiple-scale techniques to find a more general solution. The solution is uniformly valid throughout the boundary layer and the interior regions of the shell.

The two perturbation parameters used in the analysis reflect, separately, the influence of the edge load and the shell's geometric properties on the approximations to the solution. If the load-dependent perturbation parameter is set equal to zero, the solution reduces to Hildebrand's [6] linear approximation of the problem. If the terms associated with the geometrically-dependent perturbation parameter are set equal to zero and an expansion made near the loaded edge, the solution is Reissner's boundary-layer approximation [9] of the problem.

2. Governing differential equations. The differential equations governing the finite axisymmetric deformation of a variable-thickness circular cylindrical shell acted upon by edge loads are [4]

$$
\begin{gathered}
\beta^{\prime \prime}+\left(D^{\prime} / D\right) \beta^{\prime}+(\cos \beta-1) \sin \beta+\nu\left(D^{\prime} / D\right)(1-\cos \beta)=-(a / D) \Psi \cos \beta \\
\Psi^{\prime \prime}-\left(C^{\prime} / C\right) \Psi^{\prime}-\left[\sin ^{2} \beta+\nu\left\{\beta^{\prime} \cos \beta-\left(C^{\prime} / C\right) \sin \beta\right\}\right] \Psi=a C \sin \beta
\end{gathered}
$$

in the absence of axial loading. In these equations the primes denote differentiation with respect to the dimensionless axial coordinate

$$
\xi=z / a
$$

shown in Fig. 1. Also, $\Psi$ is a stress function, $\beta$ is the rotation experienced by a shell generator, $\nu$ is Poisson's ratio, $a$ is the radial distance to the shell's midsurface and

$$
D=\frac{E h^{3}}{12\left(1-\nu^{2}\right)}, \quad C=E h
$$

are the flexural and extensional rigidity, respectively, where $h$ is the thickness at a given point $\xi$ and $E$ is Young's modulus. If $\Psi$ and $\beta$ are known, the stress resultants, stress couples, and radial displacement can be found from the following:

$$
\begin{gathered}
a N_{\xi}=\Psi \sin \beta, \quad a Q=-\Psi \cos \beta, \quad a N_{\theta}=\Psi^{\prime}, \\
a M_{\xi}=D\left[\beta^{\prime}+\nu(1-\cos \beta)\right], \quad a M_{\theta}=D\left[(1-\cos \beta)+\nu \beta^{\prime}\right], \\
E h u=\Psi^{\prime}-\nu \Psi \sin \beta .
\end{gathered}
$$

The positive directions of the various quantities are shown in Fig. 1.

Introducing the dimensionless variables

$$
t(\xi)=\frac{h(\xi)}{h_{0}}, \quad \psi(\xi)=\frac{\Psi(\xi)}{A}, \quad \alpha(\xi)=\frac{\beta(\xi)}{\delta},
$$

it is possible to study the influence of loading and shell dimensions on approximations 


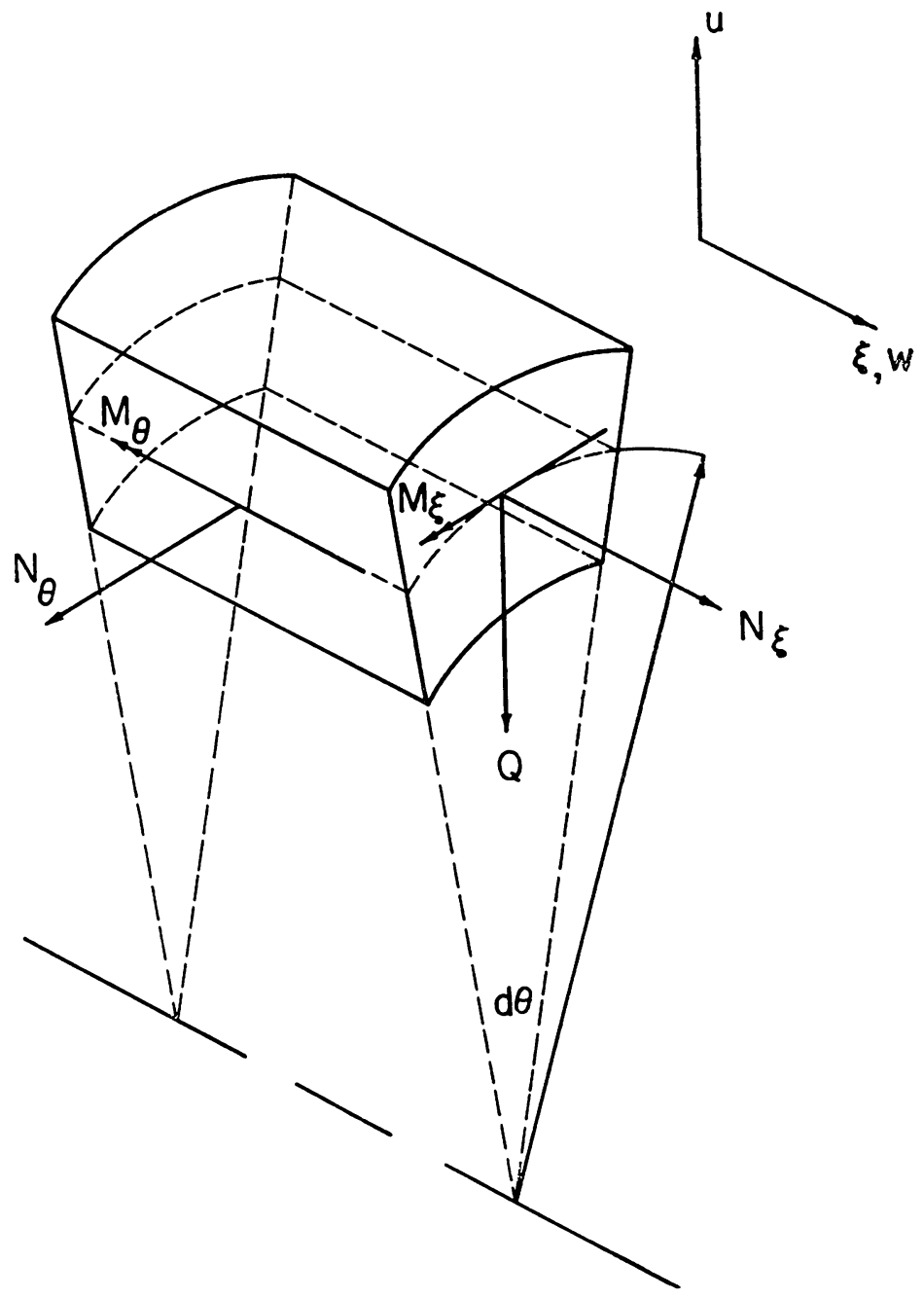

FIG. 1. Element of shell with stress resultants and stress couples.

to the governing differential equations. In the above equations $t, \psi$ and $\alpha$ are functions of order one, $h_{0}$ is the thickness at the loaded edge, and $A$ and $\delta$ are constants formulated in the course of the analysis. Upon substituting Eqs. (7) into Eqs. (1) the governing differential equations become

$$
\begin{array}{r}
\alpha^{\prime \prime}+f \alpha^{\prime}+\frac{1}{\delta}[\cos (\delta \alpha)-1] \sin (\delta \alpha)+\frac{\nu}{d}[1-\cos (\delta \alpha)] f=-\frac{\lambda^{2}}{d} \psi \cos (\delta \alpha), \\
\psi^{\prime \prime}-P \psi^{\prime}-\left[\sin ^{2}(\delta \alpha)+\nu\left\{\delta \alpha^{\prime} \cos (\delta \alpha)-P \sin (\delta \alpha)\right\}\right] \psi=t L^{2} \sin (\delta \alpha),
\end{array}
$$

where

$$
\begin{aligned}
f & =3\left(t^{\prime} / t\right), \quad d=t^{3}, & P & =t^{\prime} / t, \\
\lambda^{2} & =12\left(1-\nu^{2}\right) \frac{a A}{\delta E h_{0}^{3}}, & L^{2} & =\frac{a E h_{0}}{A} .
\end{aligned}
$$


If the deformations are only moderately large, the sines and cosines in Eqs. (8) are represented to a sufficient degree of accuracy by the first few terms of a Taylor series, and the governing equations for the cylinder are then

$$
\begin{aligned}
& \alpha^{\prime \prime}+f \alpha^{\prime}+\frac{\nu}{2} \delta \alpha^{2} f-\frac{\delta^{2}}{2} \alpha^{3}+\cdots=-\frac{\psi}{\tau^{2} d}\left[1-\frac{\delta^{2}}{2} \alpha^{2}+\cdots\right], \\
& \psi^{\prime \prime}-P \psi^{\prime}- {\left[\delta \nu \left\{\left(1-\frac{\delta^{2}}{2} \alpha^{2}+\cdots\right) \alpha^{\prime}\right.\right.} \\
&\left.\left.-P\left(\alpha-\frac{\delta^{2}}{6} \alpha^{3}+\cdots\right)\right\}+\delta^{2} \alpha^{2}\right] \psi=\frac{t}{\tau^{2}}\left[\alpha-\frac{\delta^{2}}{6} \alpha^{3}+\cdots\right],
\end{aligned}
$$

where

$$
1 / \tau^{2}=\lambda^{2}=L^{2} \delta
$$

The latter relationship is necessary to produce a consistent set of boundary-layer equations at the loaded edge and yields, upon substitution of Eqs. (10),

$$
\tau^{4}=h_{0}^{2} / 12\left(1-\nu^{2}\right) a^{2} \ll 1
$$

which, on the basis of thin shell theory, is a small parameter.

3. Edge-bending moment load on a semi-infinite cylinder. If a semi-infinite circular cylinder is acted upon by an edge-bending moment of magnitude $M_{0}$, as shown in Fig. 2, the boundary conditions are found by considering Eqs. (4) which give

$$
a M_{0}=D(0)\left[\delta \alpha^{\prime}(0)+\nu\{1-\cos (\delta \alpha(0))\}\right], \quad 0=\psi(0),
$$

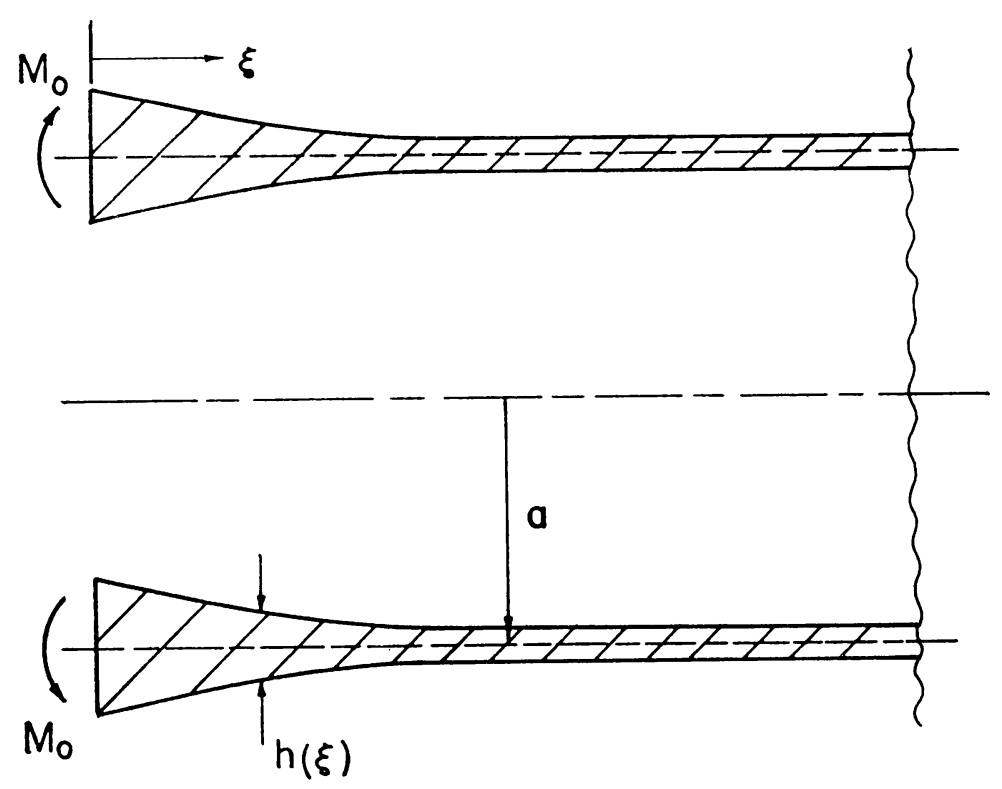

Fia. 2. Variable-thickness cylinder with moment load $M_{0}$. 
along with the requirement of finiteness of $\psi$ and $\alpha$ as $\xi \rightarrow \infty$. The boundary conditions for moderately large deformations, corresponding to Eqs. (11), are therefore

$$
a M_{0}=\delta D(0)\left[\alpha^{\prime}(0)+(\nu \delta / 2) \alpha^{2}(0)+\cdots\right], \quad 0=\psi(0) .
$$

3.1. Moderately large deformation solution by the multiple-scale technique. Assuming the deformations are only moderately large (i.e., $\delta$ small), closed-form approximations are found for a semi-infinite circular cylinder with edge-bending moments by using the method of multiple scales. Following the work on the linear equations [1] the new independent variables

$$
\eta=g(\zeta) / \tau, \quad \zeta=\xi
$$

are introduced such that

$$
\psi(\xi)=\psi(\zeta, \eta), \quad \alpha(\xi) \equiv \alpha(\zeta, \eta) .
$$

This transforms the ordinary differential equations into a set of partial differential equations where the asymptotic representations

$$
\begin{aligned}
& \psi \sim \psi_{00}(\zeta, \eta)+\tau \psi_{01}(\zeta, \eta)+\delta \tau \psi_{11}(\zeta, \eta)+\cdots \\
& \alpha \sim \alpha_{00}(\zeta, \eta)+\tau \alpha_{01}(\zeta, \eta)+\delta \tau \alpha_{11}(\zeta, \eta)+\cdots
\end{aligned}
$$

are assumed so that each approximation is bounded independent of $\tau$ and $\delta$. A class of variable-thickness circular cylinders is treated by retaining the variable coefficients and assuming that the thickness function $t$ and its derivatives are continuous and of order one.

By substituting Eqs. (16)-(18) into Eqs. (11) and equating to zero coefficients of like combinations of $\delta^{i} \tau^{j}$, the following systems of partial differential equations result: First-order problems $\left(1 / \tau^{2}\right.$ terms $)$ :

$$
\left(g^{\prime}\right)^{2} \alpha_{00 \eta \eta}=-\left(\psi_{00} / d\right), \quad\left(g^{\prime}\right)^{2} \psi_{00 \eta \eta}=t \alpha_{00} .
$$

Second-order problems $(1 / \tau$ terms $)$ :

$$
\begin{aligned}
\left(g^{\prime}\right)^{2} \alpha_{01 \eta \eta}+\left\{2 g^{\prime} \alpha_{00 \zeta \eta}+\left(g^{\prime \prime}+f g^{\prime}\right) \alpha_{00 \eta}\right\} & =-\left(\psi_{01} / d\right), \\
\left(g^{\prime}\right)^{2} \psi_{01 \eta \eta}+\left\{2 g^{\prime} \psi_{00 \zeta \eta}+\left(g^{\prime \prime}-P g^{\prime}\right) \psi_{00 \eta}\right\} & =t \alpha_{01} .
\end{aligned}
$$

Third-order problems $\left(\delta^{\circ} \tau^{\circ}, \delta^{2} / \tau^{2}\right.$ and $\delta / \tau$ terms $)$ :

$$
\begin{gathered}
\left(g^{\prime}\right)^{2} \alpha_{02 \eta \eta}+\left\{2 g^{\prime} \alpha_{01 \zeta \eta}+\left(g^{\prime \prime}+f g^{\prime}\right) \alpha_{01 \eta \zeta}+\alpha_{00 \zeta \zeta}+f \alpha_{00 \zeta}\right\}=-\left(\psi_{02} / d\right) \\
\left(g^{\prime}\right)^{2} \psi_{02 \eta \eta}+\left\{2 g^{\prime} \psi_{01 \zeta \eta \zeta}+\left(g^{\prime \prime}-P g^{\prime}\right) \psi_{01 \lambda}+\psi_{00 \zeta \zeta}-P \psi_{00 \zeta}\right\}=t \alpha_{02} \\
\left(g^{\prime}\right)^{2} \alpha_{20}=-\left(\psi_{20} / d\right)+\left(\psi_{00} / 2 d\right) \alpha_{00}^{2} \\
\left(g^{\prime}\right)^{2} \psi_{20}=t \alpha_{20}-(t / 6) \alpha_{00}^{3} \\
\left(g^{\prime}\right)^{2} \alpha_{11 \eta \eta}+\left\{2 g^{\prime} \alpha_{10 \zeta \eta}+\left(g^{\prime \prime}+f g^{\prime}\right) \alpha_{10 \eta}\right\}=-\left(\psi_{11} / d\right) \\
\left(g^{\prime}\right)^{2} \psi_{11 \eta \eta}+\left\{2 g^{\prime} \psi_{10 \zeta \eta}+\left(g^{\prime \prime}-P g^{\prime}\right) \psi_{10 n}\right\}=v g^{\prime} \alpha_{00 \eta} \psi_{00}+t \alpha_{11} .
\end{gathered}
$$

Fourth-order problems $\left(\delta, \delta^{2} / \tau\right.$ terms):

$$
\left(g^{\prime}\right)^{2} \alpha_{12 \eta \eta}+\left\{2 g^{\prime} \alpha_{11 \zeta \eta}+\left(g^{\prime \prime}+f g^{\prime}\right) \alpha_{11 \eta}\right\}+(\nu / 2) f \alpha_{00}^{2}=-\left(\psi_{12} / d\right),
$$




$$
\begin{gathered}
\left(g^{\prime}\right)^{2} \psi_{12 \eta \eta}+\left\{2 g^{\prime} \psi_{11 \zeta \eta}+\left(g^{\prime \prime}-P g^{\prime}\right) \psi_{11 \eta}\right\}-\nu g^{\prime} \alpha_{01 \eta} \psi_{00}-\nu g^{\prime} \psi_{01} \alpha_{00 \eta} \\
-\nu \alpha_{00 \zeta} \psi_{00}-\nu P \alpha_{00} \psi_{00}=t \alpha_{12}, \\
\left(g^{\prime}\right)^{2} \alpha_{21 \eta \eta}+\left\{2 g^{\prime} \alpha_{20 \zeta \eta}+\left(g^{\prime \prime}+f g^{\prime}\right) \alpha_{20 \eta}\right\}=-\left(\psi_{21} / d\right)+\psi_{00}^{2} \alpha_{01}+\left(\psi_{01} / 2\right) \alpha_{00}^{2} \\
\left(g^{\prime}\right)^{2} \psi_{21 \eta \eta}+\left\{2 g^{\prime} \psi_{20 \zeta \eta}+\left(g^{\prime \prime}-P g^{\prime}\right) \psi_{20 \eta}\right\}=t \alpha_{21}+\cdots
\end{gathered}
$$

and so on. A subscript $\eta$ or $\zeta$ denotes partial differentiation with respect to the corresponding variable. Boundary conditions for each of the above problems are found in like manner and are

$$
\begin{gathered}
0=\psi_{i j}(0,0), \\
1=g^{\prime}(0) \alpha_{00 \eta}(0,0), \quad 0=g^{\prime}(0)_{\alpha 01 \eta}(0,0)+\alpha_{00 \zeta}(0,0), \\
0=\alpha_{20 \eta}(0,0), \quad 0=g^{\prime}(0) \alpha_{11 \eta}(0,0)+(\nu / 2) \alpha_{00}^{2}(0,0)+\alpha_{10 \zeta}(0,0),
\end{gathered}
$$

and so on, together with the requirement that $\psi$ and $\alpha$ are bounded as $\eta$ goes to infinity. The first-order boundary condition, Eq. (27a), was normalized without any loss in generality, producing the additional relationship

$$
\tau=\delta D(0) / a M_{0}
$$

between the constants $\tau$ and $\delta$ which, upon substitution into Eqs. (10) and (12), gives

$$
\begin{gathered}
\delta^{2}=\left(a M_{0}^{2} / h_{0}^{5} E^{2}\right)\left[12\left(1-\nu^{2}\right)\right]^{3 / 2}, \\
A=M_{0}\left[12\left(1-\nu^{2}\right)\right]^{1 / 4}\left(a / h_{0}\right)^{1 / 2} \\
\tau^{4}=\left(1 / 12\left(1-\nu^{2}\right)\right)\left(h_{0}^{2} / a^{2}\right)
\end{gathered}
$$

The terms in the braces in Eqs. (19)-(25) result in secular terms which are removed on the basis of Lighthill's principle [1].

3.1.1. Solution of the first-order problem. The first-order problem for a circular cylindrical shell loaded by an edge-bending moment is solved by considering Eqs. (19), (26) and (27) in conjunction with Lighthill's principle. By separation of variables the stress function is of the form

$$
\psi_{00}=Y(\eta) Z(\zeta)
$$

which, upon substitution into Eq. (19), yields

$$
Y^{\prime \prime \prime \prime}+4 \mu^{4} Y=0
$$

where the constant

$$
\mu^{4}=\frac{1}{4}\left(t /\left(g^{\prime}\right)^{4} d\right)
$$

is set equal to one with no loss in generality. Keeping only bounded solutions, Eqs. (19), (26), and the above result in the first-order solutions

$$
\psi_{00}=B_{00}(\zeta) e^{-\eta} \sin \eta, \quad \alpha_{00}=-\left(B_{00}(\zeta) / t^{2}\right) e^{-\eta} \cos \eta
$$

where $\eta$ is given by (16a); then from Eqs. (9b) and (32) we find

$$
g=\frac{1}{\sqrt{ } 2} \int_{0}^{\xi} \frac{d \zeta}{t^{1 / 2}}
$$


This expression for the boundary layer variable is consistent with the solution of the linear deformation problem [1]. The coefficient $B_{00}$ must remove the secular terms from Eqs. (20) and, therefore, is governed by the differential equation

$$
2 g^{\prime} B_{00}^{\prime}+\left(g^{\prime \prime}+f g^{\prime}\right) B_{00}=0 .
$$

Thus

$$
B_{00}=b_{00} t^{3 / 4},
$$

where $b_{00}$ is a constant determined by the boundary condition (27a) which demands that $b_{00}=\sqrt{ } 2$.

3.1.2. Solutions of higher-order problems. The solutions of the higher-order problems progress in the same manner as the above. For example, since the first-order solution removed the secular terms in Eqs. (20), the second-order solutions are

$$
\psi_{01}=B_{01}(\zeta) e^{-\eta} \sin \eta, \quad \alpha_{01}=-\left(B_{01}(\zeta) / t^{2}\right) e^{-\eta} \cos \eta .
$$

Then, upon introduction of Eq. (21a) into (21b) and removal of secular terms, it is found that the following must be true:

$$
0=2\left[2 g^{\prime} t\left(\frac{\psi_{01}}{t^{2}}\right)_{\zeta \eta}+\frac{5}{2} \frac{g^{\prime} t^{\prime}}{t^{2}} \psi_{01 \eta}\right]-\frac{5}{4} \frac{1}{t^{2}}\left(t^{\prime} \psi_{00}\right)_{5}+\frac{3}{4}\left(\frac{t^{\prime} \psi_{00}}{t^{2}}\right)_{\zeta}
$$

The equation has been simplified by using Eqs. (9) and (19). Substituting Eqs. (33a) and (37a) into Eq. (38) and noting that the relationship so obtained must hold for arbitrary $\eta$ then yields

$$
B_{01}=t^{3 / 4}\left\{\frac{b_{00} \sqrt{ } 2}{4} \int_{0}^{\xi}\left[\frac{1}{4} \frac{t^{\prime \prime}}{t^{1 / 2}}+\frac{15}{16} \frac{\left(t^{\prime}\right)^{2}}{t^{3 / 2}}\right] d \zeta+b_{01}\right\} ;
$$

the constant $b_{01}$ is found from the boundary condition (27b).

3.2. Summary of the multiple-scale solution. To find the remaining terms in the multiple-scale solution of the problem of a semi-infinite circular cylinder loaded by an edge-bending moment load of magnitude $M_{0}$ the above steps are repeated for Eqs. (22)(25) with the boundary conditions (26) and (27). The resulting four-term asymptotic solutions are then

$$
\begin{aligned}
& \Psi=A \psi \sim A\left[\psi_{00}+\tau \psi_{01}+\delta \tau \psi_{11}+\delta^{2} \psi_{20}\right] \\
& \beta=\delta \alpha \sim \delta\left[\alpha_{00}+\tau \alpha_{01}+\delta \tau \alpha_{11}+\delta^{2} \alpha_{20}\right]
\end{aligned}
$$

where

$$
\psi_{0 i}=B_{0 i} e^{-\eta} \sin \eta, \quad \alpha_{0 i}=-\left(B_{0 i} / t^{2}\right) e^{-\eta} \cos \eta .
$$

for $i=0,1$,

$$
\begin{aligned}
& \psi_{11}=\left[A_{11} \cos \eta+B_{11} \sin \eta\right] e^{-\eta}+\frac{\nu \sqrt{ } 2}{5}\left[1+\frac{2}{3}\{\cos 2 \eta+\sin 2 \eta\}\right] e^{-2 \eta}, \\
& \alpha_{11}=\frac{1}{t^{2}}\left[A_{11} \sin \eta-B_{11} \cos \eta\right] e^{-\eta}-\frac{\nu \sqrt{ } 2}{10 t^{2}}\left[1-\frac{1}{3}\{\sin 2 \eta-\cos 2 \eta\}\right] e^{-2 \eta}, \\
& \psi_{20}=\left[A_{20} \cos \eta+B_{20} \sin \eta\right] e^{-\eta}+\frac{B_{00}^{3}}{160 t^{3}}[4 \cos \eta-2 \sin \eta-\sin 3 \eta] e^{-3 \eta},
\end{aligned}
$$




$$
\begin{gathered}
\alpha_{20}=\frac{1}{t^{2}}\left[A_{20} \sin \eta-B_{20} \cos \eta\right] e^{-\eta}+\frac{B_{00}^{3}}{160 t^{3}}\left[\frac{7}{3} \cos 3 \eta+2 \cos \eta+4 \sin \eta\right] e^{-3 \eta}, \\
\eta=\frac{1}{\tau \sqrt{ } 2} \int_{0}^{\xi} \frac{d \zeta}{t^{1 / 2}} \\
B_{00}=\sqrt{ } 2 t^{3 / 4} \\
B_{01}=t^{3 / 4}\left\{\frac{1}{8} \int_{0}^{\xi}\left[\frac{t^{\prime \prime}}{t^{1 / 2}}+\frac{15}{4} \frac{\left(t^{\prime}\right)^{2}}{t^{3 / 2}}\right] d \zeta-\frac{5}{2} t^{\prime}(0)\right\} \\
A_{11}=-\nu(\sqrt{ } 2 / 3) t^{3 / 4}, \quad B_{11}=-5 \nu(\sqrt{ } 2 / 3) t^{3 / 4} \\
A_{20}=-(\sqrt{ } 2 / 20) t^{3 / 4}, \quad B_{20}=(13 / 80) \sqrt{ } 2 t^{3 / 4}
\end{gathered}
$$

and the constants $\tau, A$ and $\delta$ are as given in Eqs. (29). The solution implies that beyond the second approximation the corrections to the first-order solution are relatively small even for reasonably large $\delta$.

The stress resultants and displacements can be found by substituting directly from Eqs. (40) into Eqs. (4)-(6).

4. Extension of analysis. To alter the solution for general boundary conditions such as $M_{\xi}(0)=M_{\iota} ; Q(0)=Q_{\iota}$ and for pressurization $(P)$ the expansion of Eqs. (18) would be replaced by

$$
\begin{aligned}
& \psi \sim \sum_{i=0}^{\infty} \sum_{i=0}^{\infty} \sum_{k=0}^{\infty} \sum_{l=0}^{\infty} \delta^{i} \tau^{i} \epsilon^{k} \Delta^{l} \psi_{i k j l}, \\
& \alpha \sim \sum_{i=0}^{\infty} \sum_{i=0}^{\infty} \sum_{k=0}^{\infty} \sum_{l=0}^{\infty} \delta^{i} \tau^{i} \epsilon^{k} \Delta^{l} \alpha_{i j k l} .
\end{aligned}
$$

The solution would proceed as above with $\delta, \epsilon$ and $\Delta$ being dependent upon the loadings $M_{\epsilon}, Q_{e}$ and $P$, respectively. An expansion like Eqs. (49) would then lead to a general study of how the loading, variable thickness, and thickness-to-curvature ratio $(\tau)$ affect the approximations.

5. Conclusion. For a circular cylindrical shell with a symmetric edge-bending moment load the analysis has produced a uniformly valid four-term asymptotic solution for finite deformation without relaxing the provision for thickness changes. The variables $A_{i j}, B_{i j}$ and $\eta$ reflect this provision and contain corrections necessary to make the solution valid both in the boundary layer (i.e., near $\xi=0$ ) and in the interior of the shell.

The solution, Eqs. (40)-(48), contains Hildebrand's [6] linear solution $(\delta=0)$ and Reissner's [9] nonlinear constant-thickness boundary-layer approximation $(\tau=0, \xi \rightarrow 0)$ as special cases. As expected, the linear portion of the expansion is load-insensitive.

The nonlinear corrections $\psi_{11}$ and $\alpha_{11}$ are present even for constant-thickness circular cylindrical shells and, although they are neglected in Reissner's boundary layer solution [9] for thin shells, there can be certain parametric combinations for which they would be significant.

The $\delta^{2}$-terms of the expansion, Eqs. (40), are small for most problems and can be neglected.

To gain an appreciation for the relative magnitude of the corrections to the constantthickness linear theory solution, consider the rotation $\beta$ at the loaded edge $(\xi=0)$. 
From Eqs. (40)-(48) it follows that upon neglecting $\delta^{2}$-terms:

$$
\beta(0)=\delta \sqrt{ } 2\left[-1+(5 \sqrt{ } 2 / 4) t^{\prime}(0) \tau-(25 \nu / 3) \tau \delta\right] .
$$

It can be seen that for small finite deformation the $\tau$-term would become significant when the edge thickness-to-curvature ratio approaches the limit between thin and thick shellsan upper limit on the magnitude of $\tau$ is about 0.15 and the thickness change $\left(t^{\prime}\right)$ is of order one at $\xi=0$. The $\tau \delta$-term representing the transition from linear theory to nonlinear theory becomes significant when the loading becomes large $(\delta \rightarrow 1)$.

\section{References}

[1] M. Van Dyke, Perturbation methods in fluid dynamics, 1st edition, Academic Press, New York, 1969

[2] J. A. Cochran, Problems in singular perturbation theory, Tech. Rep. No. 1, Applied Mathematics and Statistics Lab., Stanford University, 1962

[3] J. R. Stafford, A multiple scale solution for circular cylindrical shells, Int. J. Solids Structures 5, 855-861 (1969)

[4] E. Reissner, On axisymmetrical deformations of thin shells of revolution, Proc. Symp. Appl. Math. 3, 27-52 (1949), McGraw-Hill

[5] J. D. Cole and J. Kevorkian, Uniformly validasymptotic approximations for certain nonlineardifferential equations, in Nonlinear differential equations and nonlinear mechanics, J. P. LaSalle and S. Lefchetz, eds., pp. 113-120, Academic Press, New York, 1963

[6] F. B. Hildebrand, On asymptotic integration in shell theory, Proc. Symp. Appl. Math. 3, 53-66 (1949), McGraw-Hill

[7] T. J. Lardner, Symmetric deformation of a circular cylindrical shell of variable thickness, Zeit. Ange. Math. Phys. 19, 270-277 (1968)

[8] E. Reissner and J. H. Weinitschke, Finite pure bending of circular cylindrical tubes, Quart. Appl. Math. 20, 305-319 (1963)

[9] E. Reissner, On influence coefficients and nonlinearity for thin shells of revolution, J. Appl. Mech. 26, 69-72 (1959) 